\title{
Analysis of Reverse-Bias Leakage Current Mechanisms in Metal/GaN Schottky Diodes
}

\author{
P. Pipinys and V. Lapeika \\ Department of Physics, Vilnius Pedagogical University, 08106 Vilnius, Lithuania \\ Correspondence should be addressed to V. Lapeika, vytautas.lapeika@vpu.lt \\ Received 13 May 2010; Accepted 28 July 2010 \\ Academic Editor: Jörg Fink
}

Copyright ( $\odot 2010$ P. Pipinys and V. Lapeika. This is an open access article distributed under the Creative Commons Attribution License, which permits unrestricted use, distribution, and reproduction in any medium, provided the original work is properly cited.

\begin{abstract}
Temperature-dependent reverse-bias current-voltage characteristics obtained by other researchers for Schottky diodes fabricated on $\mathrm{GaN}$ are reinterpreted in terms of phonon-assisted tunneling (PhAT) model. Temperature dependence of reverse-bias leakage current is shown could be caused by the temperature dependence of electron tunneling rate from traps in the metal-semiconductor interface to the conduction band of semiconductor. A good fit of experimental data with the theory is received in a wide temperature range (from $80 \mathrm{~K}$ to $500 \mathrm{~K}$ ) using for calculation the effective mass of $0.222 \mathrm{~m}_{\mathrm{e}}$. and for the phonon energy the value of $70 \mathrm{meV}$. The temperature and bias voltages dependences of an apparent barrier height (activation energy) are also explicable in the framework of the PhAT model.
\end{abstract}

\section{Introduction}

$\mathrm{GaN}$ is a wide direct band gap semiconductor which has unique applications in the fabrication of blue light-emitting diodes, lasers, ultraviolet detectors, field effect transistors, and high power rectifiers $[1,2]$. Metal-semiconductor contact is one of the most widely used rectifying contacts in the electronics industry. However, GaN-based Schottky contacts suffer from abnormal leakage currents under reverse bias, which is one factor presently limiting device performance.

Due to the technological importance of Schottky diodes, a full understanding of the nature of their electrical characteristics is of great interest. Recently a number of papers on reverse-bias leakage current mechanisms in M/GaN Schottky diodes appeared [3-16]. In papers [17-20], the investigation of Schottky contact on GaN nanowires were presented.

Suggestions of investigators about the reverse-bias leakage current mechanism are very different. For instance, Miller et al. [3] claimed that two dominant leakage current mechanisms in Ni/n-GaN Schottky diode (SD) fabricated on $\mathrm{GaN}$ grown by molecular-beam epitaxy can be identified. One associated with field-emission tunneling and another with an exponential temperature dependence, consistent with either trap-assisted tunneling or one-dimensional hopping conduction. However, for the fitting of the experimental data with the tunneling current model, the authors [3] have used unphysically low the effective mass $(9.8 \times$ $10^{-3} \mathrm{~m}_{\mathrm{e}}$ ) and the value for the effective Richardson's constant $A^{*}\left(0.001 \mathrm{~A} / \mathrm{cm}^{2} \mathrm{~K}^{2}\right)$. Zhang et al. [4] analyzed the leakage current mechanisms in the Schottky contacts of both $\mathrm{n}-\mathrm{GaN}$ and $\mathrm{AlGaN} / \mathrm{GaN}$ epitaxial layer structures at different temperatures and concluded that tunneling current dominates at temperatures below $150 \mathrm{~K}$ whereas the Frenkel-Poole emission dominates at temperatures higher than $250 \mathrm{~K}$. Huang et al. on the basis of current-voltage measurements between 27 and $350^{\circ} \mathrm{C}$ in $\mathrm{Au} / \mathrm{Ni} / \mathrm{GaN}$ SDs concluded that thermionic-emission model with a Gaussian distribution of barrier heights is responsible for the electrical behavior of the diodes at temperatures lower than $230^{\circ} \mathrm{C}$, while the generation-recombination process takes place at temperatures above $230^{\circ} \mathrm{C}$ [7]. Arslan et al. [11] assumed that the reverse-bias leakage current mechanisms in SDs on $\mathrm{Al}_{0.83} \operatorname{In}_{0.17} \mathrm{~N} / \mathrm{AlN} / \mathrm{GaN}$ heterostructures, in the temperature range of $250-375 \mathrm{~K}$ is based on the Frenkel-Poole emission model. Moreover, Iucolano et al. [13] claimed that onedimensional variable-range hopping conduction was one of the dominant carrier transport mechanisms for the Ni/GaN Schottky sample annealed at $1150^{\circ} \mathrm{C}$ for 5 minutes. 
A such variety of proposed conduction mechanisms involved to describe the leakage current in $\mathrm{M} / \mathrm{GaN}$ diodes implies that fundamental properties of the conduction mechanisms are not to date fully understood.

Another problem which emerges in examining the practical diodes characteristics is observed dependence of the barrier height derived from $I-V / T$ measurements on applied bias voltage and temperatures. In terms of a classical thermionic emission theory [21] the forward current density is given by

$$
j=j_{0}\left[\exp \left(\frac{q V}{n k T}\right)-1\right]
$$

where

$$
j_{0}=A^{*} T^{2} \exp \left(-\frac{q \Phi_{\mathrm{BO}}}{k T}\right)
$$

is the saturation current density, $A^{*}$ is the effective Richardson constant, $\Phi_{\mathrm{BO}}$ is the Schottky barrier height $(\mathrm{SBH}), k$ is the Boltzmann constant, $q$ is the electronic charge unit, and $n$ is the ideality factor which describes the deviation of practical diodes from the pure thermionic-emission model characterized by $n=1$.

A so-called Richardson plot of $\ln \left(j_{0} / A^{*} T^{2}\right)$ versus inverse temperature $1 / T$ is often used to determine the $\mathrm{SBH}$ from the slope of the thermally activated behavior. According to (2)

$$
\ln \left(\frac{j_{0}}{A^{*} T^{2}}\right)=-\frac{q \Phi_{\mathrm{BO}}}{k T}
$$

and the plot $\ln \left(j_{0} / A^{*} T^{2}\right)$ versus $1 / T$ (Richardson plot) should yield a straight line with an activation energy $E_{T}=$ $-q \Phi_{\mathrm{BO}}$ if the $\mathrm{SBH} \Phi_{\mathrm{BO}}$ is temperature dependent. But in most cases these plots do not yield straight lines (see e.g., $[15,16])$. The curving in these plots results in (determines) dependence of $E_{T}$ on temperature.

In the case of reverse bias voltage when $(q V / k T) \gg 1$ for the current density jr from (2) one obtains

$$
j_{r}=A^{*} T^{2} \exp \left(-\frac{q \Phi_{\mathrm{br}}}{k T}\right) .
$$

The apparent SBH $\Phi_{\text {br }}$ calculated from the gradient of the Richardson plot lines will be lower than $\Phi_{\mathrm{BO}}$ due to the lowering of the barrier height caused by image-force, but temperature dependence of both $\Phi_{\mathrm{BO}}$ and $\Phi_{\mathrm{br}}$ has the same shape [14].

Analysis of the $I-V / T$ characteristics of Schottky diodes on the basis of thermionic emission theory reveals a decrease in the apparent barrier height $\Phi_{\mathrm{BO}}$ and an increase in the ideality factor $n$ with a decrease in temperature $[5,6,12$, $15,16,19]$. The temperature dependence of the apparent barrier height is frequently explained by invoking a Gaussian distribution of the SBH values at the interface between the Schottky metal and the semiconductor $[15,16,19]$.

In previous work [22], we have shown that in $\mathrm{Ni} / \mathrm{n}-\mathrm{GaN}$ $\mathrm{SD}$ the leakage current dependence on temperature in a wide range of temperatures can be described in the framework of phonon-assisted tunneling (PhAT) model. The purpose of this work is on the basis of this model to explain the peculiarities of the reverse-bias current dependence on bias voltage and their temperature behavior in the M/GaN Schottky diodes presented by various authors in recent publications. Dependence of the apparent SBH on temperature discussed by many investigators is also explained in the framework of the PhAT model.

\section{Theory and Comparison with Experimental Data}

2.1. The Phonon-Assisted Tunneling Model. In accordance with phonon-assisted tunneling model, the current transport through the barrier is governed by a process of tunneling from states nearby metal-semiconductor interface to the conduction band of the semiconductor. The electron population in the states is assumed to be independent of bias voltage due to the continuous filling of interface states from the metallic electrode (see Figure 1). If the electrons released from these centers dominate the current through the diode, the current density, neglecting scattering of electrons by phonons and recombination process, will be equal to

$$
I_{r}=q N_{S} W S,
$$

where $N_{S}$ is the charged states density at the interface, $S$ is the area of barrier electrode, and $W$ is the electron tunneling rate from these states into the conduction band which is a function of field strength $E$ and temperature $T$. Therefore, $I_{r} \approx W(E, T)$, and we can fit the current dependences obtained by measurements with the theoretical tunnel transition through the barrier rate dependences on temperature.

The electrons tunneling rate $W(E, T)$ from centers of $\varepsilon_{T}$ depth at the metal/semiconductor interface is $[22,23]$

$$
\begin{gathered}
W(E, T)=\frac{q E}{\left(8 m^{*} \varepsilon_{T}\right)^{1 / 2}}\left[\left(1+\gamma^{2}\right)^{1 / 2}-\gamma\right]^{1 / 2}\left[1+\gamma^{2}\right]^{-1 / 4} \\
\times \exp \left\{-\frac{4}{3} \frac{\left(2 m^{*}\right)^{1 / 2}}{q E \hbar} \varepsilon_{T}^{3 / 2} \times\left[\left(1+\gamma^{2}\right)^{1 / 2}-\gamma\right]^{2}\right. \\
\left.\times\left[\left(1+\gamma^{2}\right)^{1 / 2}+\frac{1}{2} \gamma\right]\right\}, \\
\gamma=\frac{(2 m *)^{1 / 2} \Gamma^{2}}{8 q \hbar E \varepsilon_{T}^{1 / 2}} .
\end{gathered}
$$

Here, $\Gamma^{2}=\Gamma_{0}^{2}(2 n+1)=8 a(\hbar \omega) 2(2 n+1)$ is the width of the centre absorption band caused mainly by interaction with optical phonons of energy $\hbar \omega, n=[\exp (\hbar \omega / k T)-1]^{-1}$ is the temperature distribution of phonons, $m^{*}$ is the effective density mass of the electron in the semiconductor, and $a$ is the electron-phonon coupling constant. The parameter $\gamma$ provides the temperature dependence for tunneling process. If $\gamma$ is very small due to a very large electrical field or low temperature, the tunneling process is temperature independent, and in this case PhAT is similar to classical tunneling. 


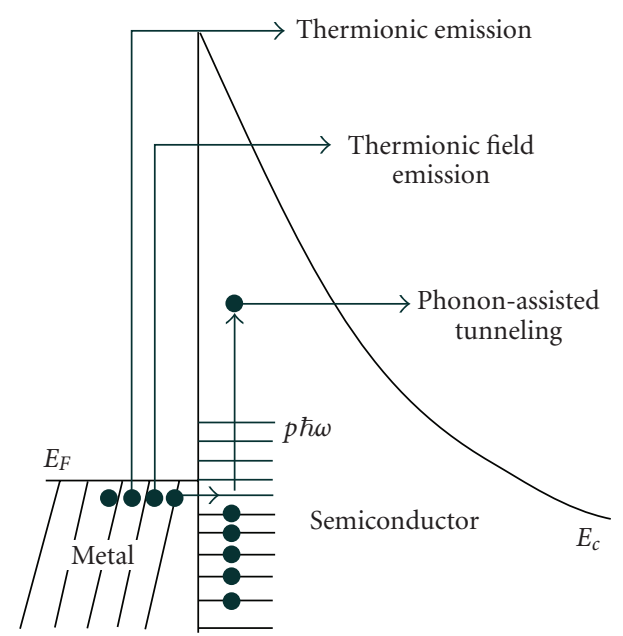

FIGURE 1: Energy diagram of the metal/semiconductor contact.

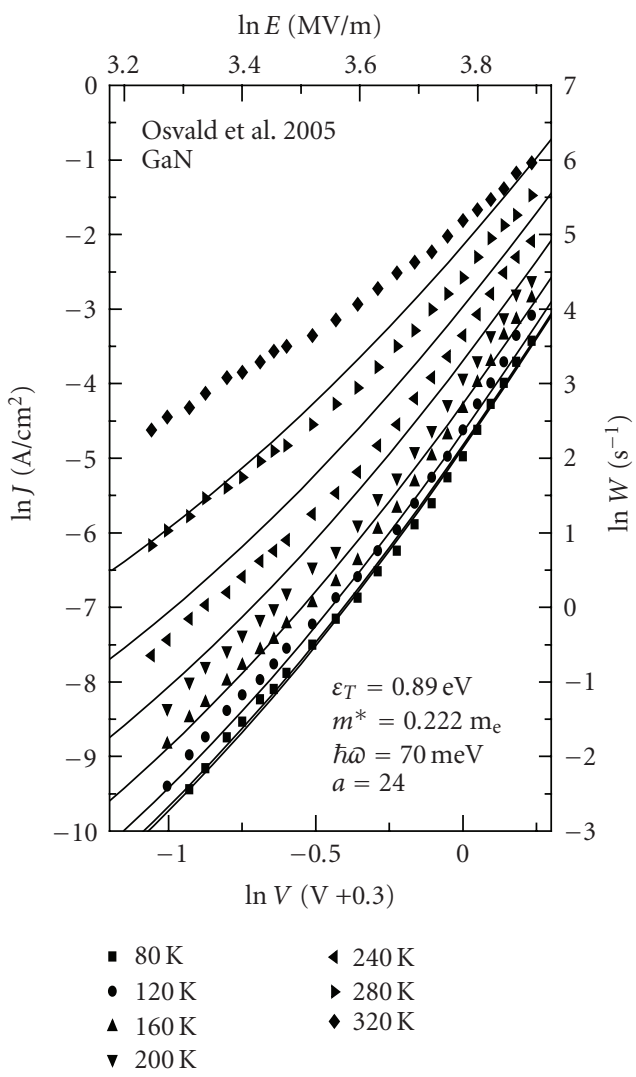

Figure 2: Comparison of $I-V$ dependences for metal/n-GaN Schottky diode from Figure 2 [5] (symbols) with theoretical $W(E, T)$ against $E$ dependences (solid curves), computed using parameters: $\varepsilon_{T}=0.89 \mathrm{eV}, m^{*}=0.222 \mathrm{~m}_{\mathrm{e}}, \hbar \omega=70 \mathrm{meV}, a=2.4$.

\subsection{The Comparison of the Leakage Current Dependences} on Bias for Different Temperatures with Theoretical W(E,T) Dependences. Now let us represent the comparison of some $I-V / T$ characteristics measured on diodes fabricated both on epitaxial GaN and on GaN nanowires with the PhAT rate dependence on field strength computed using (6). The fit of

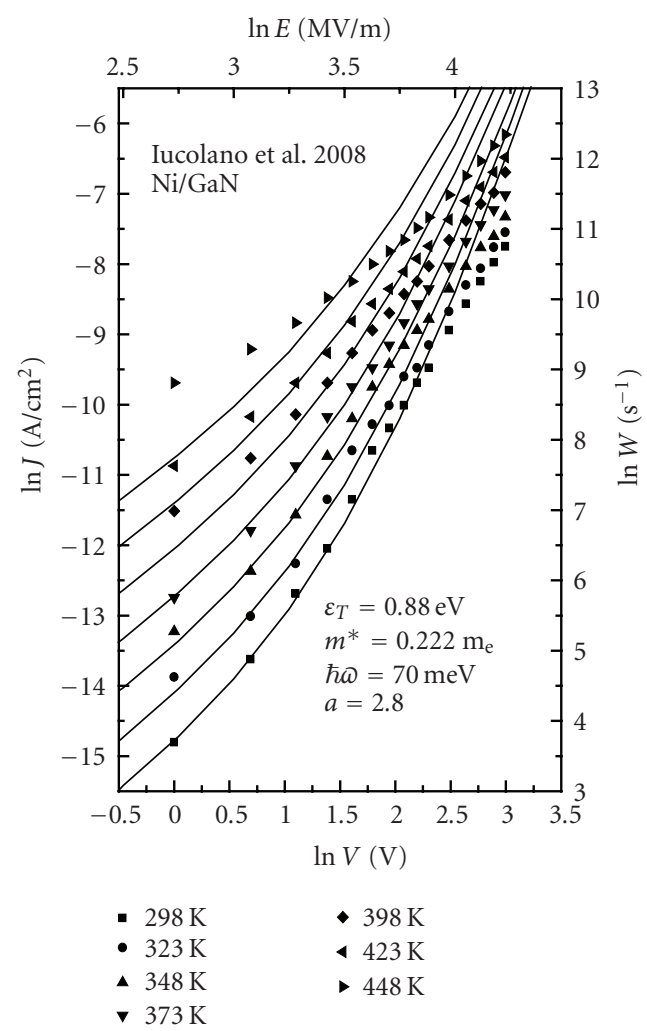

Figure 3: The $I-V$ characteristics of Ni/GaN diode extracted from Figure 6 in [13] (symbols) fitted to the theory (solid curves). Parameters for computation: $\varepsilon_{T}=0.88 \mathrm{eV}, m^{*}=0.222 \mathrm{~m}_{\mathrm{e}}, \hbar \omega=$ $70 \mathrm{meV}, a=2.8$. Estimated states density $N_{s}=6.2 \times 10^{10} \mathrm{~cm}^{-2}$.

the leakage $I-V / T$ curves obtained by Osvald et al. [5] in the temperature range from 80 to $320 \mathrm{~K}$ for metal/n-GaN diode fabricated on $\mathrm{N}$-polarity GaN grown by molecular-beam epitaxy to the tunneling rate dependences on field strength $W(E, T)$ is shown in Figure 2. The fit is performed under the assumption that the field strength is proportional to the square root of applied voltage, that is, the tunneling occurs in the high field region. The theoretical $W(E, T)$ versus $E$ dependences were computed using for the barrier height $\varepsilon_{T}$ the value of $0.89 \mathrm{eV}$ estimated from the $C-V$ data [5], and for the electron effective mass the the value of $0.222 \mathrm{~m}_{\mathrm{e}}$ [24] was used. For the phonon energy the value of $70 \mathrm{meV}$ (i.e., somewhat lower than the energy of LO phonon, which in $\mathrm{GaN}$ is equal to $91 \mathrm{meV}[25]$ ) was selected. The coupling constant $a$ was chosen in order to get the best fit of the experimental data with the calculated dependences. As is seen in Figure 2, the theoretical $W(E, T)$ dependences fit reasonably well with the experimental data for entire range of the measured temperatures. It is possible to notice that the higher the reverse bias is, the weaker is the temperature dependence. The estimated density of charged states in the interface was found to be equal to $1.5 \times 10^{15} \mathrm{~cm}^{-2}$.

Figure 3 shows the $I-V / T$ data measured by Iucolano et al. [13] for Ni/GaN Schottky diode made on epitaxial n-GaN layer fitted to the theoretical $W(E, T)$ curves. As is seen from Figure 3, the theoretical curves describe well the 


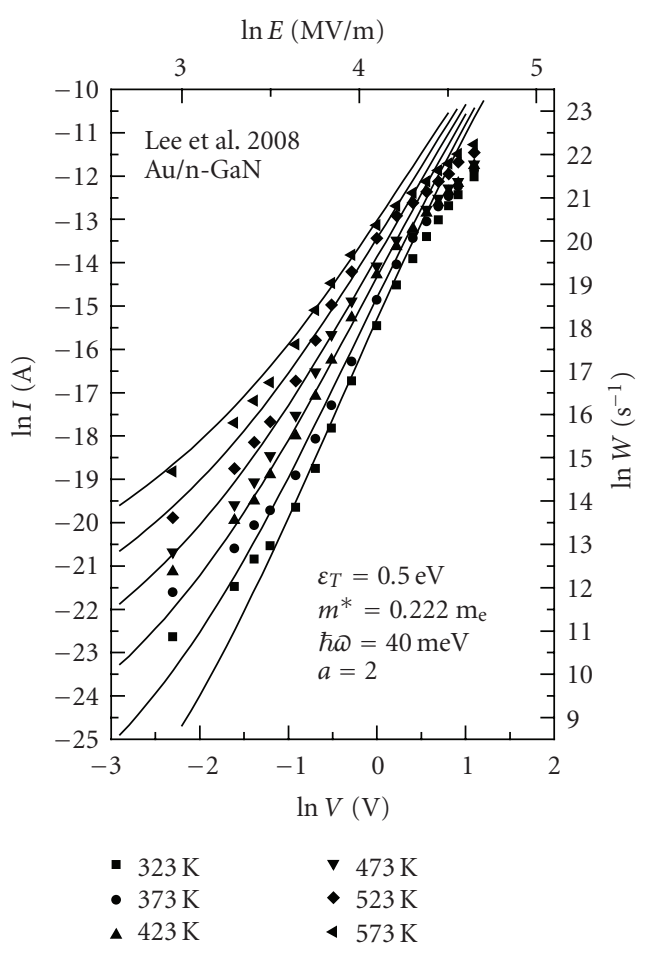

Figure 4: The $I-V$ characteristics of Au/n-GaN nano-Schottky diode represented from Figure 2 [19] (symbols) fitted to theoretical $W(E, T)$ against $E$ dependences (solid curves). Parameters for computation: $\varepsilon_{T}=0.5 \mathrm{eV}, m^{*}=0.222 \mathrm{~m}_{\mathrm{e}}, \hbar \omega=40 \mathrm{meV}, a=2.0$.

experimental data in the temperature range from $298 \mathrm{~K}$ to $448 \mathrm{~K}$. Only the $I-V$ curve measured at $448 \mathrm{~K}$ signally deviates from theoretical ones. Other discrepancy between the theory and experiment emerges at higher voltages/fields when the current approaches to saturation. The observed trend to saturation of the current at higher electric field could be due to limitation of the filled centers in the interface when their exhaustion rate is very high (approximately $10^{10} \mathrm{~s}^{-1}$ ). The assessed traps density for this diode was found to be equal to $6 \times 10^{10} \mathrm{~cm}^{-2}$.

$I-V / T$ characteristics for $\mathrm{Au} / \mathrm{n}-\mathrm{GaN}$ nano-Schottky diode measured by Lee et al. [19] in the temperature range from 323 to $573 \mathrm{~K}$ are shown in Figure 4 . The temperature behavior of the $I-V$ curves are very similar to the curves in Figure 3 and are also well described by the PhAT model. The electrical characteristics of a single GaN nanowire Schottky diode by authors of [19] were explained by a thermionic-field emission and an enhancement of the tunneling effects.

In a very recent paper, the temperature-dependent $I$ $V$ characteristics of Pt Schottky contacts to a-plane ntype $\mathrm{GaN}$ were presented [26]. A notable deviation from the theoretical Richardson constant value was observed in the conventional Richardson plot. The authors of [26] have concluded that the thermionic emission model is inapplicable for these diodes. To explain the observed electrical behaviors, defect-assisted tunneling was necessary to invoke.

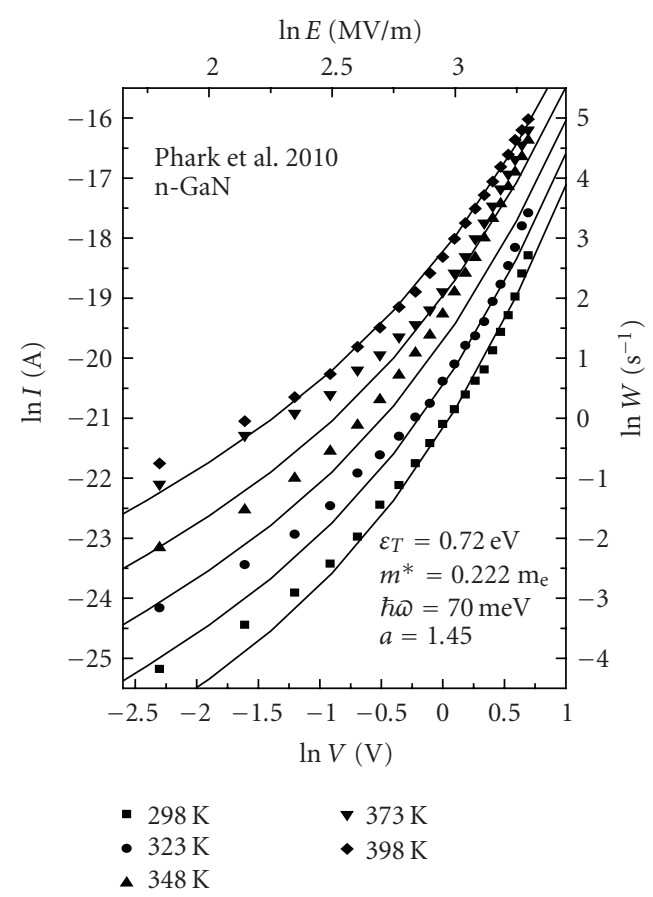

Figure 5: Reverse bias $I-V$ characteristics of the Pt Schottky contacts to $a$-plane n-GaN extracted from Figure 2 [26] (symbols) fitted to theory (solid curves) Parameters: $\varepsilon_{T}=0.72 \mathrm{eV}, m^{*}=$ $0.222 \mathrm{~m}_{\mathrm{e}}, \hbar \omega=70 \mathrm{meV}, a=1.45$. Assessed state density $N_{S}=1.4$. $10^{13} \mathrm{~cm}^{-2}$.

In Figure 5, the fit of the reverse bias branch of $I_{r}$ V/T characteristics from Figure 2 [26] with PhAT model is exposed. As can be seen the theoretical curves describe well the experimental data for all measured temperatures using the same value of $\varepsilon_{T}=0.72 \mathrm{eV}$ assessed in [26]. From the fit of the experimental data with the theory and using (5) ( $\mathrm{S}=$ $4.2 \times 10^{-4} \mathrm{~cm}^{2}$ ) estimated states density at the interface was found to be equal to $1.4 \times 10^{13} \mathrm{~cm}^{-2}$.

\subsection{Apparent Barrier Height Dependence on Temperature. In} this section, we present a comparison of the apparent barrier height dependence on temperature with envisaged for such dependence by PhAT model.

The reason for SBH derived from $I-V / T$ characteristics depends on temperature lies in the fact that $\Phi_{\mathrm{br}}$ is calculated from the gradient of Richardsons' plots which, in general, are not straight lines. The steepness of $\ln \left(I_{R} / T^{2}\right)$ versus $1 / T$ curves at higher voltages was found to decline at lower temperatures, and the bowing of these curves is observed $[15,16]$. The phonon-assisted theory predicts, namely, the same value of decrease in $\Phi_{b r}$ as obtained from $I-V / T$ characteristics. The correctness of this assertion will be confirmed by the comparison of the theoretical activation energy $E_{T}$ dependences upon temperature with the apparent barrier height dependence on the same parameter. $E_{T}$ was 


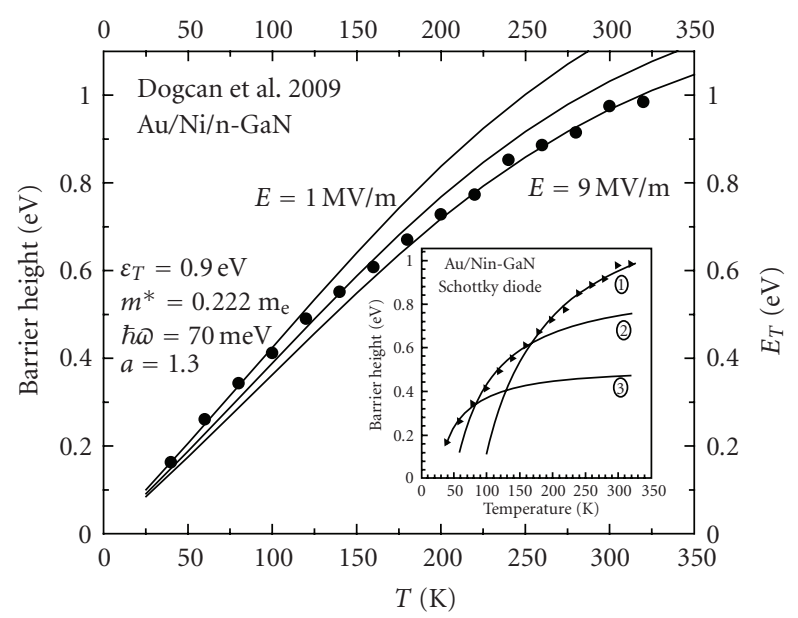

Figure 6: Temperature dependence of the barrier height for $\mathrm{Au} / \mathrm{Ni} / \mathrm{n}-\mathrm{GaN}$ from [16] (symbols), fitted to the theoretical $E_{T}$ versus $T$ (solid lines) computed for field strengths of 1, 5, $9(\mathrm{MV} / \mathrm{m})$. Note that theoretical $E_{T}$ is higher at lower fields. The inset shows barrier height versus $T$ from the same work. The continuous curves in the inset correspond to $\Phi_{b}$ computed by authors of [16] using TE model for the three Gaussian distributions of the barrier height.

calculated as the gradient of theoretical "Richardon plot" using

$$
E_{T}=\frac{k}{q} \frac{d \ln \left(W / T^{2}\right)}{d(1 / T)} .
$$

In Figure 6 the apparent SBH dependence on temperature assessed by Doğan et al. for Au/Ni/n-GaN Schottky diodes from references [16] fitted to $E_{T}$ dependence on temperature is shown. As is seen in Figure 6 the theoretical $E_{T}$ versus $T$ dependence reflects well the apparent barrier height dependence on temperature. An abnormal temperature dependence of the barrier height the authors of [16] have explained by invoking three sets of Gaussian distributions of barrier heights at 320-160 K, 160-80 K, and $80-40 \mathrm{~K}$ (see inset of Figure 6).

Figure 7 shows the fit of temperature-dependent $\mathrm{SBH}$ for $\mathrm{Pd} / \mathrm{Au} / \mathrm{n}-\mathrm{GaN}$ from [12] and $\mathrm{Ag} / \mathrm{p}-\mathrm{GaN} / \mathrm{SDs}$ [14] with the theoretical $E_{T}$ versus $T$ dependence. One can see that theoretical curves both for n-type and p-type GaN describe very well the strong temperature dependence of apparent SBH observed for these diodes. Such the SBH behavior in [12] was attributed to barrier inhomogeneities by assuming a Gaussian distribution of barrier heights at the interface. Temperature variation of the experimental value of the SBH derived from the reverse bias characteristics from $0.17 \mathrm{eV}$ at $80 \mathrm{~K}$ to $0.84 \mathrm{eV}$ at $360 \mathrm{~K}$ for the $\mathrm{Ag} / \mathrm{p}-\mathrm{GaN}$ SD; the authors of [14] ascribed to the charge transport tunneling mechanism.

It is worth mentioning that the strong temperature dependence of the apparent Schottky barier height is also peculiarity for SDs made on the basis of other semiconductors. For instance in Figure 8 we represent the temperature dependence of $\mathrm{SBH}$ assessed from $I-V / T$ data for $\mathrm{SD}$ made on n-type Si [27] and on p-type Si [28] ones. As can be seen the theoretical curves $E_{T}$ versus $T$ computed using

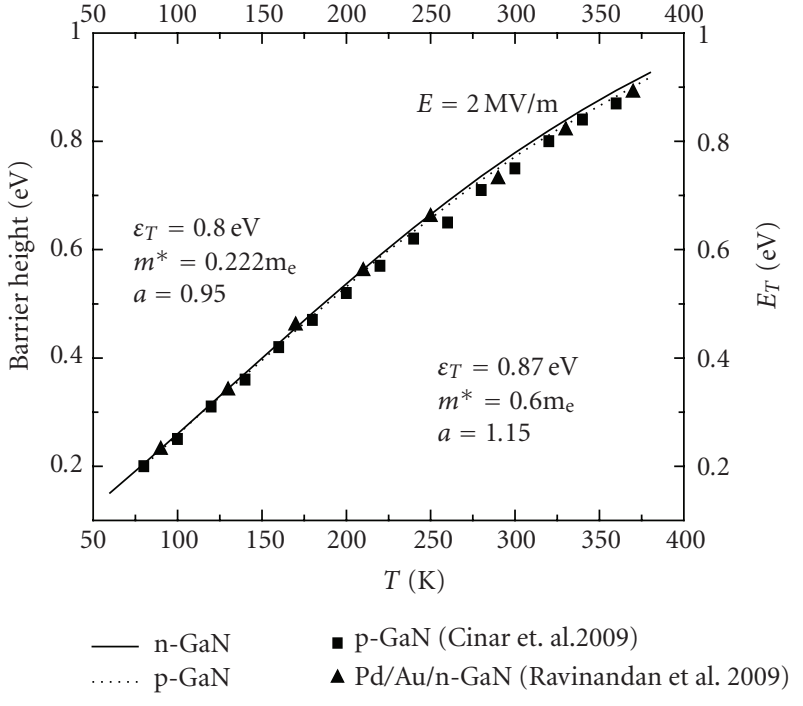

FIGURE 7: Variation of the barrier height with temperature for $\mathrm{Pd} / \mathrm{Au} / \mathrm{n}-\mathrm{GaN}$ from [12] and Ag/p-GaN [14] (symbols), fitted to the $E_{T}$ versus $T$ exposed by solid line for $n$-type and dot line for p-type GaN.

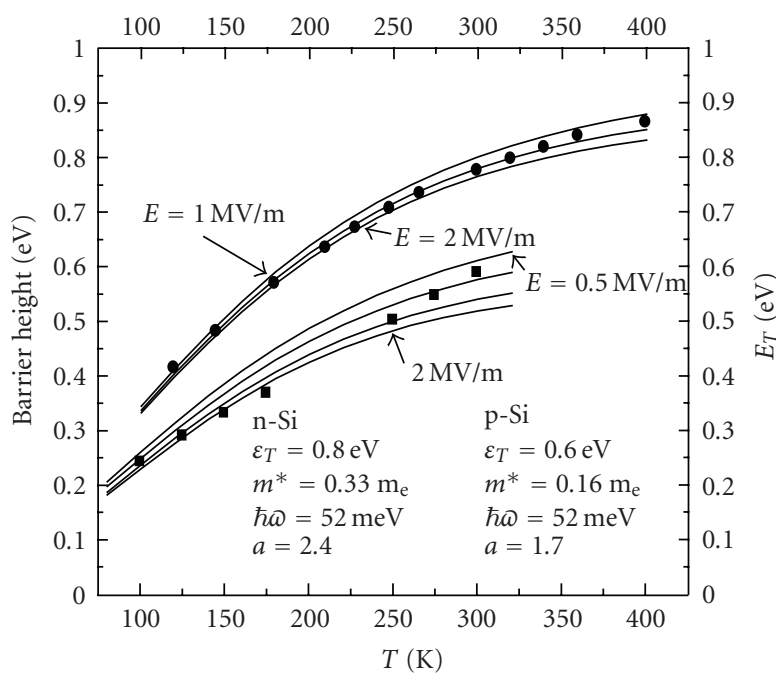

- Faruk Yuksel et al. Al/p-Si

- Rodrigues. n-Si

Figure 8: Temperature dependence of the barrier height for nSi from [26] and Al/p-Si [27] symbols, fitted to the $E_{T}$ versus $T$ dependences (solid lines).

(6) and (7) and inherent for silicon parameters describe the experimental $\mathrm{SBH}$ dependence on temperature very well. The temperature dependence of the apparent SBH in these SDs by authors of $[27,28]$ have been explained invoking the Schottky barrier height inhomogeneity model [29]. Under their opinion, in presence of inhomogeneities of SBH electrons at low temperatures are able to surmount the lower barriers, and therefore the current transport will be dominated by current flowing through the patches of lower Schottky barrier height. As the temperature increases, 
an increasing number of electrons will have sufficient energy to surmount the higher barriers; consequently, the dominant barrier height will increase with increasing both temperature and bias voltage. However, actual decrease in barrier height with increasing bias is observed for many diodes (see, e.g., [30] and references therein). We want to emphasize that PhAT theory predicts the $\mathrm{SBH}$ decrease as a bias voltage increases. This assertion is proved by $E_{T}$ dependence on temperature computed for different values of field strength (see Figures 6 and 8 ).

The physical essence of the apparent barrier height dependence both on temperature and bias voltage in the framework of the PhAT theory is comprehensible. According to this model, the apparent SBH/(activation energy) depends on the quantity of phonons taken part in the tunneling process. At low temperatures the number of phonons according to the equation $n=[\exp (\hbar \omega / k T)-1]^{-1}$ will be less in determining lower energy activation and herewith barrier height. At higher temperatures the number of phonons is greater, and apparent $\mathrm{SBH}$ will be higher. Likewise is explicable dependence of $\mathrm{SBH}$ on bias voltage. At high voltages for the tunneling less quantity of phonons is required then at low ones. Hence, at low bias voltages the apparent SBH will be higher. Thus, the actual barrier height can be determined from the $C-V$ measurements but not from $I-V / T$ measurements using thermionic emission theory.

\section{Conclusion}

In conclusion, the phonon-assisted tunneling model describes well the peculiarities of reverse-bias currenttemperature dependence in Schottky diodes fabricated either on GaN epitaxial layer or on GaN nanowires. The fit of experimental data to computed tunneling rate allows to estimate the field strength at which the free charge carriers are generated, and the density of charged states near the interface between metal and semiconductor. In the terms of this model the bias voltage and temperature dependence of apparent SB height evaluated from $I-V / T$ measurements is explained as well. Thus, phonon-assisted tunneling mechanism must be taken into account in explaining the reverse leakage current characteristics for diodes with Schottky barriers.

\section{References}

[1] S. C. Jain, M. Willander, J. Narayan, and R. Van Overstraeten, "III-nitrides: growth, characterization, and properties," Journal of Applied Physics, vol. 87, no. 3, pp. 965-1006, 2000.

[2] O. Ambacher, "Growth and applications of group III-nitrides," Journal of Physics D, vol. 31, no. 20, pp. 2653-2710, 1998.

[3] E. J. Miller, E. T. Yu, P. Waltereit, and J. S. Speck, "Analysis of reverse-bias leakage current mechanisms in GaN grown by molecular-beam epitaxy," Applied Physics Letters, vol. 84, no. 4, pp. 535-537, 2004.

[4] H. Zhang, E. J. Miller, and E. T. Yu, "Analysis of leakage current mechanisms in Schottky contacts to GaN and $\mathrm{Al}_{0.25} \mathrm{Ga}_{0.75} \mathrm{~N} / \mathrm{GaN}$ grown by molecular-beam epitaxy," Journal of Applied Physics, vol. 99, no. 2, Article ID 023703, 6 pages, 2006.
[5] J. Osvald, J. Kuzmik, G. Konstantinidis, P. Lobotka, and A. Georgakilas, "Temperature dependence of GaN Schottky diodes I-V characteristics," Microelectronic Engineering, vol. 81, no. 2-4, pp. 181-187, 2005.

[6] Y.-J. Lin, "Electronic transport and Schottky barrier heights of $\mathrm{Pt} / n$-type GaN Schottky diodes in the extrinsic region," Journal of Applied Physics, vol. 106, no. 1, Article ID 013702, 4 pages, 2009.

[7] S. Huang, B. Shen, M. J. Wang et al., "Current transport mechanism of AuNiGaN Schottky diodes at high temperatures," Applied Physics Letters, vol. 91, no. 7, Article ID 072109, 3 pages, 2007.

[8] H. Hasegawa and S. Oyama, "Mechanism of anomalous current transport in n-type GaN Schottky contacts," Journal of Vacuum Science and Technology B, vol. 20, no. 4, pp. 16471655, 2002.

[9] J. W. P. Hsu, M. J. Manfra, R. J. Molnar, B. Heying, and J. S. Speck, "Direct imaging of reverse-bias leakage through pure screw dislocations in GaN films grown by molecular beam epitaxy on GaN templates," Applied Physics Letters, vol. 81, no. 1, Article ID 79, p. 3, 2002.

[10] Q. Luo, J. Du, M. Yang, L. Wang, T. Jin, and Q. Yu, "Backto-back Schottky diode adopted for the measurement of GaN films and its Schottky contacts," Semiconductor Science and Technology, vol. 20, no. 6, pp. 606-610, 2005.

[11] E. Arslan, S. Bütün, and E. Ozbay, "Leakage current by Frenkel-Poole emission in $\mathrm{Ni} / \mathrm{Au}$ Schottky contacts on $\mathrm{Al}_{0.83} \mathrm{In}_{0.17} \mathrm{~N} / \mathrm{AlN} / \mathrm{GaN}$ heterostructures," Applied Physics Letters, vol. 94, no. 14, Article ID 142106, 3 pages, 2009.

[12] M. Ravinandan, P. K. Rao, and V. R. Reddy, "Analysis of the current-voltage characteristics of the Pd/Au Schottky structure on n-type GaN in a wide temperature range," Semiconductor Science and Technology, vol. 24, no. 3, Article ID 035004, 2009.

[13] F. Iucolano, F. Roccaforte, F. Giannazzo, and V. Raineri, "Influence of high-temperature GaN annealed surface on the electrical properties of Ni/GaN Schottky contacts," Journal of Applied Physics, vol. 104, no. 9, Article ID 093706, 2008.

[14] K. Çınar, N. Yıldırım, C. Coşkun, and A. Turut, "Temperature dependence of current-voltage characteristics in highly doped Ag/p-GaN/In Schottky diodes," Journal of Applied Physics, vol. 106, no. 7, Article ID 073717, 2009.

[15] M. Mamor, "Interface gap states and Schottky barrier inhomogeneity at metal/n-type GaN Schottky contacts," Journal of Physics Condensed Matter, vol. 21, no. 33, Article ID 335802, 2009.

[16] S. Doǧan, S. Duman, B. Gürbulak, S. Tüzemen, and H. Morkoç, "Temperature variation of current-voltage characteristics of $\mathrm{Au} / \mathrm{Ni} / \mathrm{n}-\mathrm{GaN}$ Schottky diodes," Physica E, vol. 41, no. 4, pp. 646-651, 2009.

[17] J.-R. Kim, H. Oh, H. M. So et al., "Schottky diodes based on a single GaN nanowire," Nanotechnology, vol. 13, no. 5, pp. 701704, 2002.

[18] S.-Y. Lee and S.-K. Lee, "Current transport mechanism in a metal-GaN nanowire Schottky diode," Nanotechnology, vol. 18, no. 49, Article ID 495701, 2007.

[19] S.-Y. Lee, C.-O. Jang, J.-H. Hyung, T.-H. Kim, and S.-K. Lee, "High-temperature characteristics of GaN nano-Schottky diodes," Physica E, vol. 40, no. 10, pp. 3092-3096, 2008.

[20] C. Hwang, J.-H. Hyung, S.-Y. Lee et al., "The formation and characterization of electrical contacts (Schottky and Ohmic) on gallium nitride nanowires," Journal of Physics D, vol. 41, no. 10, Article ID 105103, 2008.

[21] E. H. Rhoderick and R. H. Williams, Metal-Semiconductor Contacts, Clarendon Press, Oxford, UK, 2nd edition, 1988. 
[22] P. Pipinys and V. Lapeika, "Temperature dependence of reverse-bias leakage current in GaN Schottky diodes as a consequence of phonon-assisted tunneling," Journal of Applied Physics, vol. 99, no. 9, Article ID 093709, 2006.

[23] A. Kiveris, S. Kudzmauskas, and P. Pipinys, "Release of electrons from traps by an electric field with phonon participation," Physica Status Solidi (A), vol. 37, no. 1, pp. 321-327, 1976.

[24] A. M. Witowski, K. Pakuła, J. M. Baranowski, M. L. Sadowski, and P. Wyder, "Electron effective mass in hexagonal GaN," Applied Physics Letters, vol. 75, no. 26, pp. 4154-4155, 1999.

[25] V. Bougrov, M. E. Levinshtein, S. L. Rumyantsev, and A. Zubrilov, "Gallium Nitride," in Properties of Advanced Semiconductor Materials GaN, AlN, InN, BN, SiC, SiGe, M. E. Levinshtein, S. L. Rumyantsev, and M. S. Shur, Eds., pp. 1-30, John Wiley \& Sons, New York, NY, USA, 2001.

[26] S.-H. Phark, H. Kim, K. M. Song, P. G. Kang, H. S. Shin, and D.-W. Kim, "Current transport in Pt Schottky contacts to aplane n-type GaN," Journal of Physics D, vol. 43, no. 16, Article ID 165102, 2010.

[27] A. M. Rodrigues, "Extraction of Schottky diode parameters from current-voltage data for a chemical-vapor-deposited diamond/silicon structure over a wide temperature range," Journal of Applied Physics, vol. 103, no. 8, Article ID 083708, 2008.

[28] Ö. F. Yüksel, "Temperature dependence of current-voltage characteristics of Al/p-Si ( $\left.\begin{array}{lll}1 & 0 & 0\end{array}\right)$ Schottky barrier diodes," Physica B, vol. 404, no. 14-15, pp. 1993-1997, 2009.

[29] R. T. Tung, "Electron transport at metal-semiconductor interfaces: general theory," Physical Review B, vol. 45, no. 23, pp. 13509-13523, 1992.

[30] P. Pipinys, A. Pipiniene, and A. Rimeika, "Phonon-assisted tunneling in reverse biased Schottky diodes," Journal of Applied Physics, vol. 86, no. 12, pp. 6875-6878, 1999. 

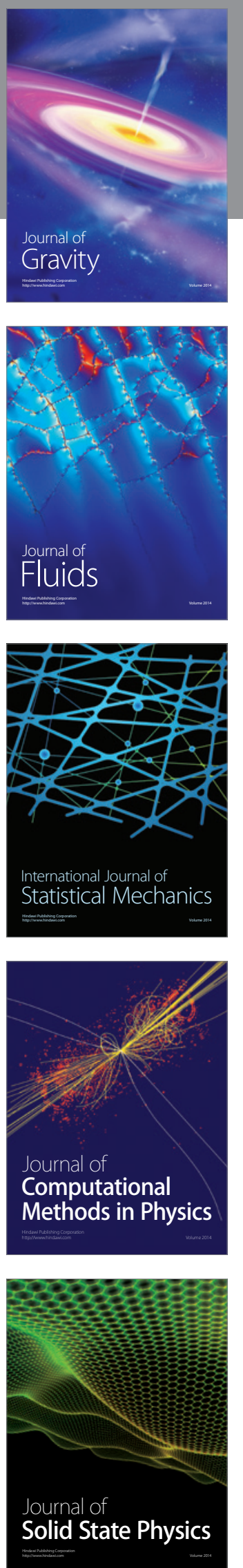

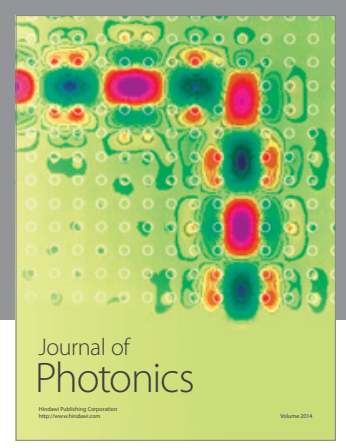

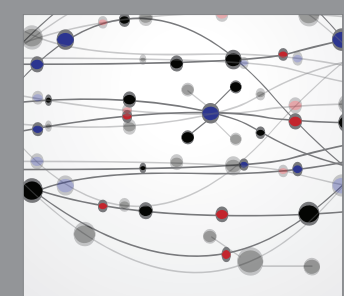

The Scientific World Journal
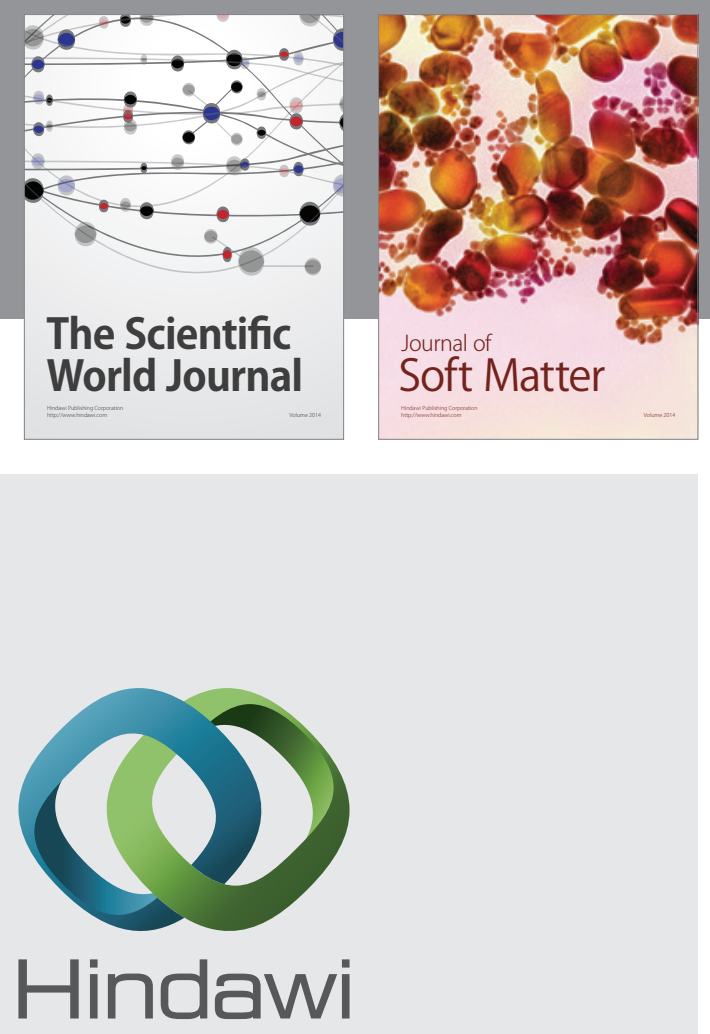

Submit your manuscripts at

http://www.hindawi.com
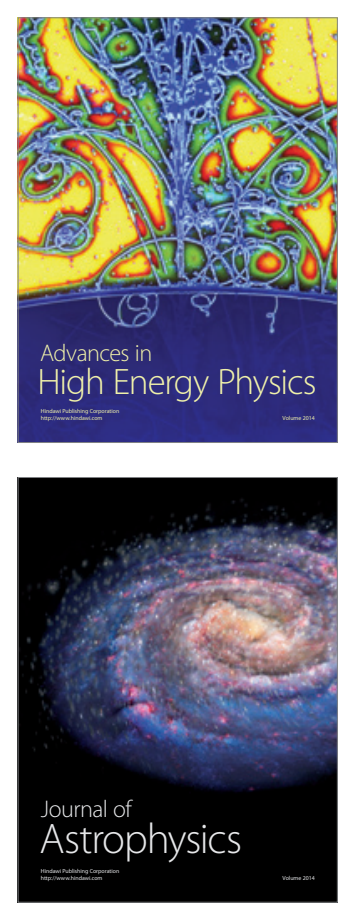
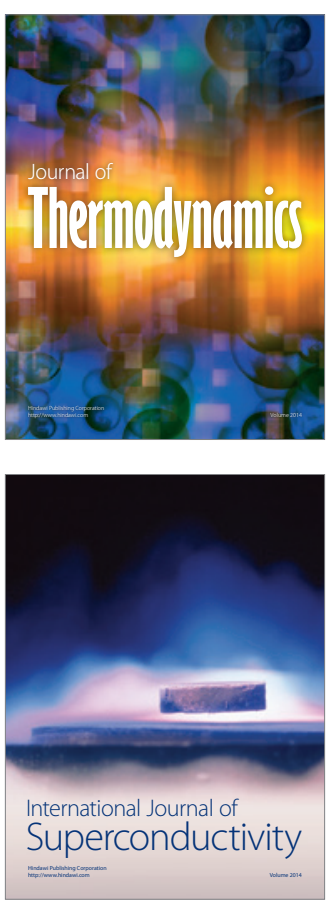
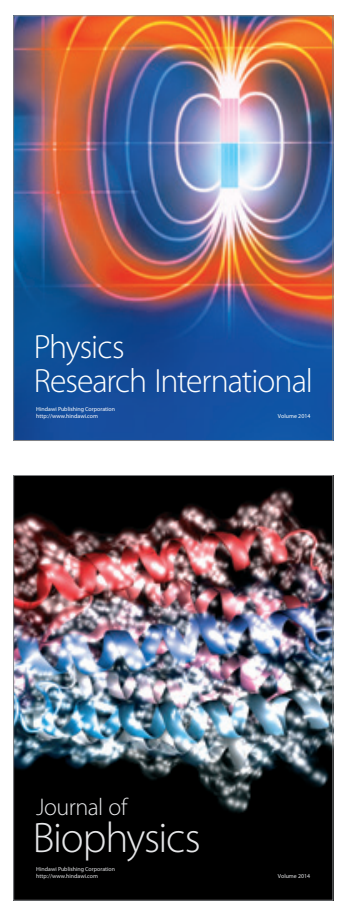
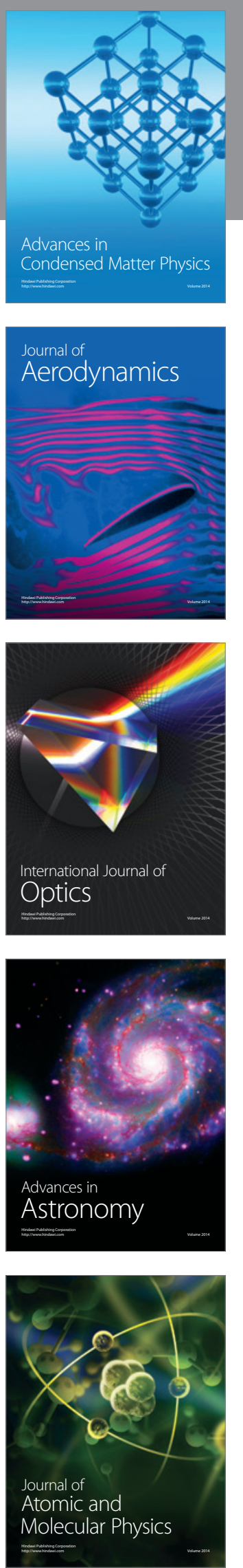